\title{
Performances agronomiques de quatre variétés de riz pluvial NERICA de plateau semées à différentes dates en zone soudano-sahélienne au Sénégal
}

\author{
Kouakou K. P-M ${ }^{1,2,3}\left(^{*}\right)$., Muller $\mathbf{B}^{3,4,5}$., Fofana $A^{6}$., Guisse $A^{1}$. \\ 1. Université Cheikh Anta Diop de Dakar, Avenue Cheikh Anta Diop, BP 5005 Dakar, Sénégal \\ 2. Institut National Polytechnique Félix Houphouët Boigny, BP 1313 Yamoussoukro, Côte d'Ivoire \\ 3. Centre d'Étude Régional pour l'Amélioration de l'Adaptation à la Sécheresse, BP 3320 Thiès-Escale, Thiès, Sénégal \\ 4. Centre du Riz pour l'Afrique, Station Sahel, BP 96 Saint-Louis, Sénégal \\ 5. Centre de Coopération Internationale en Recherche Agronomique pour le Développement, Umr Agap, F-34398 \\ Montpellier, France \\ 6. Institut Sénégalais de Recherches Agricoles, CNRA de Bambey, BP 53 Bambey, Sénégal \\ $\left(^{*}\right)$ Auteur correspondant : kkpmartial@gmail.com
}

Original submitted in on $20^{\text {th }}$ January 2016. Published online at www.m.elewa.org on $31^{\text {st }}$ March 2016 http://dx.doi.org/10.4314/jab.v99i1.4

\section{RÉSUMÉ}

Objectif : Le Sénégal est à $80 \%$ tributaire du marché international pour satisfaire sa demande intérieure en riz. Pourtant cet aliment occupe une place de premier choix dans l'alimentation quotidienne des sénégalais. Les autorités et les partenaires au développement œuvrent alors, depuis ces deux dernières décennies, à réduire la dépendance du pays vis-à-vis du riz importé à travers l'augmentation de la production nationale. Le présent travail s'inscrit dans cette dynamique. Elle s'intéresse particulièrement à l'étude, en conditions strictement pluviales, des performances agronomiques de deux variétés de riz NERICA à cycle de 75-85 jours (NERICA 8 et NERICA 11) et de deux variétés de riz NERICA à cycle de 95-100 jours (NERICA 1 et NERICA 4).

Méthodologie et résultats : Pour ce faire, deux essais ont été semés à Sinthiou Malème, pendant la saison des pluies, à des dates décalées en 2013 et répétés en 2014. Chaque année, le semis du premier essai a été effectué pendant la cinquième pentade du mois de juillet et le deuxième pendant la deuxième pentade du mois d'août. Les résultats ont montré que la saison des pluies a duré 110 jours et a été caractérisée par des périodes de déficit hydrique qui ont coïncidé avec la phase reproductive des variétés de riz étudiées. Ce déficit hydrique a perturbé la formation des panicules et la fécondation des épillets. L'effet de ce déficit hydrique a été plus prononcé sur les semis tardifs d'une part (baisse de rendement grains de 75 à $79 \%$ ) et sur les variétés à cycle plus long d'autre part (baisse de rendement grains pouvant atteindre $42 \%$ ).

Conclusion et application des résultats : Les quatre variétés de riz NERICA pluvial étudiées peuvent certes être cultivées dans la zone soudano-sahélienne de Sinthiou Malème mais il faut préférer les variétés très précoces de 75-85 jours (NERICA 8 et NERICA 11) et les semer avant la cinquième pentade du mois de juillet pour ne pas compromettre les rendements grains.

Mots-clés : date de semis, zone soudano-sahélienne, NERICA, performances agronomiques, riz pluvial, Sénégal. 


\section{Kouakou et al. J. Appl. Biosci. 2016 Performances agronomiques de quatre variétés de riz pluvial NERICA}

de plateau semées à différentes dates en zone soudano-sahélienne au Sénégal

\section{ABSTRACT}

Objective: Senegal imports every year $80 \%$ of its rice for consumption. Since the last two decades, current policies are trying to reduce the dependence of the country towards the international market by increasing the national rice production. One of the alternatives for the increase of national rice production is the development of rainfed rice in soudano-sahelian areas of the country. Therefore, the present study aimed to measure the agronomic traits of four upland rainfed rice NERICA (NERICA 1 and NERICA 4 (95-100 days); NERICA 8 and NERICA 11 (75-85 days)) in a soudano-sahelian zone.

Methodology and results: Two trials were carried out in 2013 and 2014 at Sinthiou Malème (1350' $\mathrm{N} ; 13^{\circ} 55^{\prime} \mathrm{W}$; $22 \mathrm{~m}$ ) during the raining season and no water was given through irrigation. Each year, the first trial was sown during the month of July and the second one, during the second one in the month of August. The results showed that the raining season lasted 110 days and dry spells occurred during the flowering stage of the rice varieties. Those drought periods affected negatively the panicle formation and the spikelet pollination. This phenomenon was severe on the latest trials (grain yields decreased of 75-79\%) and on the longest cycle length, varieties (grain yields decreased of $42 \%$ ).

Conclusion and application: The four rice varieties (NERICA 1, NERICA 4, NERICA 8 and NERICA 11) can be cultivated in the soudano-sahelian zone of Sinthiou Malème but it is advantageous to choose NERICA 8 and NERICA 11 for their shortest cycle length and they must been sown before the fifth decade of July.

Key words: Sowing date, soudano-sahelian zone, NERICA, agronomic traits, rainfed rice, Senegal

\section{INTRODUCTION}

Le riz est une denrée prépondérante dans l'alimentation sénégalaise tant en milieu urbain qu'en milieu rural (Kite, 1993; Kelly et al., 1992). II représente $36 \%$ du volume céréalier national consommé par an devant le mil $(26 \%)$ et le maïs (18\%) (Del Villar et al., 2011). La consommation annuelle par habitant oscille entre $80 \mathrm{~kg}$ et $100 \mathrm{~kg}$ (FAO, 2011 ; Dieng et al., 2009) et la consommation nationale est estimée à 1 million de tonnes par an (Seck, 2015 ; Brüntrup et al., 2006 ; PNAR, 2009). Malheureusement, le Sénégal est à $80 \%$ dépendant des importations pour satisfaire sa demande intérieure en riz. Ce qui représente une sortie de devises estimée à 130 milliards de FCFA par an et correspond à $16 \%$ du déficit de la balance commerciale (Seck, 2015; PNAR, 2009). Les autorités sénégalaises et les partenaires au développement ont mis en œuvre depuis ces deux dernières décennies des programmes visant à réduire la dépendance du pays vis-à-vis du riz importé (APRAO, 2012 ; ENDA DIAPOL, 2010) et à atteindre l'autosuffisance en riz en 2017. Parmi les stratégies adoptées, l'accent a été mis sur le développement de la riziculture pluviale dans les zones sud, centre et est du pays. Dans ces régions, les superficies cultivées ont connu une extension et des variétés améliorées, mises au point par la recherche agronomique, ont été distribuées aux producteurs (APRAO, 2012 ; FAO, 2011). Compte tenu de leur rusticité, de leur précocité et de leur productivité, les variétés de riz NERICA de plateau connaissent un certain succès dans le développement de la riziculture pluviale au Sénégal. Cependant ces régions, et en particulier les zones du centre et certaines parties de la zone orientale du Sénégal, sont régulièrement sujettes à des périodes de déficit hydrique dues à la date souvent aléatoire de démarrage de la saison pluvieuse, aux pauses pluviométriques au cours du cycle cultural et aux sécheresses de fin de cycle. De plus les zones centrales et orientales du Sénégal bénéficient d'un climat soudano-sahélien caractérisé par une pluviométrie annuelle variant entre $600 \mathrm{~mm}$ et 800 mm (Salack et al., 2011 ; Sarr et al., 2011) alors que l'on considère qu'il est hasardeux de cultiver du riz pluvial lorsque la pluviométrie annuelle est inférieure à $800 \mathrm{~mm}$ (Sié, 1997). L'on peut dès lors s'interroger sur les précautions à prendre dans le choix des variétés de riz et de la date de semis pour réussir la riziculture pluviale dans ces zones. La présente étude évalue les performances agronomiques de quatre variétés de riz pluvial NERICA de cycles 


\section{Kouakou et al. J. Appl. Biosci. 2016 Performances agronomiques de quatre variétés de riz pluvial NERICA de plateau semées à différentes dates en zone soudano-sahélienne au Sénégal}

contrastés semées à des dates décalées dans une zone soumise à un climat soudano-sahélien au

\section{MATÉRIELS ET MÉTHODE}

Site expérimental : Les essais ont été conduits sur la station de recherche de l'Institut Sénégalais de Recherches Agricoles de Sinthiou Malème ( $13^{\circ} 50^{\prime}$ Nord et $13^{\circ} 55^{\prime}$ Ouest, $22 \mathrm{~m}$ ). Le site de Sinthiou Malème est situé en zone soudao-sahélienne, entre les isohyètes $600 \mathrm{~mm}$ et $700 \mathrm{~mm}$ (Salack et al., 2011 ; Sié, 1997). Le climat de cette zone est caractérisé par un régime pluviométrique monomodal avec deux saisons bien contrastées. Les premières pluies sont enregistrées à partir de fin mai et la saison pluvieuse prend fin en général au cours du mois d'octobre. Cependant, seuls trois mois sont réellement humides : juillet, août et septembre. Les sols de la station de Sinthiou Malème sont sablo-argileux avec un $\mathrm{pH}$ acide $(4,95)$. Ils contiennent une faible teneur en matière organique et ont une faible capacité d'échange cationique. Ce sont donc des sols pauvres ayant un faible pouvoir de rétention hydrique. Les pentes des parcelles sont faibles laissant croire un relief plat. La végétation naturelle de la zone est dominée par un tapis herbacé abondant en saison des pluies composé en majorité de graminées, mais aussi de cypéracées et de dicotylédones.

Matériel végétal : Le matériel végétal était représenté par des variétés de riz pluvial de plateau de type NERICA (« Nouveau Riz pour l'Afrique »). Les NERICA résultent d'un croisement interspécifique entre Oryza sativa (L.), variété de riz asiatique à haut rendement et Oryza glaberrima (Steud), variété de riz originaire d'Afrique, moins productive mais mieux résistante aux stress biotiques et abiotiques des conditions africaines de culture (Angladette, 1966). Les variétés hydrides NERICA sont à la fois productives comme leur parent asiatique et résistantes comme leur parent africain (AfricaRice, 2008). Nos travaux ont porté sur quatre variétés de NERICA pluvial : NERICA 1, NERICA 4, NERICA 8 et NERICA 11. Les deux premières variétés ont un cycle moyen de 95100 jours et les deux autres sont plus précoces avec un cycle moyen de 75-85 jours. La phase végétative du cycle de NERICA 1 et de NERICA 4 dure entre 30 et 45 jours et ces variétés atteignent le stade mi-épiaison à 70 75 jours après semis. NERICA 8 et NERICA 11 ont une phase végétative d'une durée moyenne de 20-35 jours et atteignent la mi-épiaison à $55-60$ jours après semis (AfricaRice, 2008 ; Arradeau et Vergara, 1992). Parmi ces variétés, seule NERICA 1 a déjà été homologuée au
Sénégal.

Sénégal mais elle présente une plus grande sensibilité aux termites. La variété NERICA 4 est largement cultivée en milieu paysan. NERICA 11 se distingue des trois autres par son fort rendement potentiel $\left(7000 \mathrm{~kg} \cdot \mathrm{ha}^{-1}\right)$. NERICA 8 est proche de NERICA 4 du point de vue du rendement potentiel ( $\left.5000 \mathrm{~kg}_{\text {.ha- }}{ }^{-1}\right)$ et de NERICA 11 de par la durée du cycle semis-maturité (75-85 jours). Les semences utilisées au cours de ces essais provenaient de la collection d'AfricaRice Bénin.

Conduite des essais: Deux essais ont été semés, pendant la saison des pluies, à des dates décalées en 2013 et répétés en 2014. Tous ces essais ont été conduits en condition d'alimentation hydrique strictement pluviale. L'objectif initial était d'effectuer le premier semis dès l'installation de la saison des pluies, en début du mois de juillet et de semer le deuxième essai au plus tard trois semaines après le premier de sorte à ce que toutes les variétés étudiées bouclent leurs cycles avant la fin de la saison pluvieuse. Cependant, dans la pratique, le semis du premier essai de 2013 a été effectué le 22 juillet, après deux journées consécutives de pluie d'une hauteur totale de $41 \mathrm{~mm}$. Le semis du deuxième essai a eu lieu le 5 août, 3 jours après une pluie de $17 \mathrm{~mm}$. En 2014, le premier essai a été semé le 24 juillet, 5 jours après une pluie de $25 \mathrm{~mm}$. Le second essai de $2014 \mathrm{a}$ été installé le 8 août, 3 jours après une pluie de $61 \mathrm{~mm}$. Le dispositif en blocs de Fisher complètement randomisé a été retenu pour l'installation des essais. Les sites expérimentaux avaient reçu une culture d'arachide pendant l'hivernage précédant la mise en place des essais. Les essais comportaient trois répétitions en 2013 et quatre en 2014. Les parcelles élémentaires avaient une superficie de $30 \mathrm{~m}^{2}(6 \mathrm{~m} \times 5 \mathrm{~m})$ et de $16 \mathrm{~m}^{2}(4 \mathrm{~m} \times 4$ $\mathrm{m}$ ) respectivement en 2013 et en 2014. Pour chacun des quatre essais, les travaux de préparation de terrain ont consisté en un labour aux disques suivi d'un hersage. La géométrie de semis adoptée était de 0,25 $\mathrm{m}$ entre les lignes et $0,20 \mathrm{~m}$ entre les poquets d'une même ligne, soit une densité de 200000 poquets.ha-1 ${ }^{-1}$. Les plants ont été démariés à trois pieds par poquet, en condition humide, avant le tallage. Des désherbages manuels ont été effectués au besoin pour maintenir les plants en situation de non compétition avec les adventices. Des applications de carbofuran (Furadan) ont été administrées, au semis et au besoin, pour contrôler les attaques de 


\section{Kouakou et al. J. Appl. Biosci. 2016 Performances agronomiques de quatre variétés de riz pluvial NERICA de plateau semées à différentes dates en zone soudano-sahélienne au Sénégal}

déprédateurs, dont en particulier les termites. Les fertilisations chimiques ont été effectuées conformément aux recommandations de la recherche agronomique au Sénégal. II s'agissait d'un épandage d'engrais N-P-K (1515-15) à la dose de $200 \mathrm{~kg}$ ha-1 $^{-1}$ au semis et d'un apport de $150 \mathrm{~kg} \cdot$ ha $^{-1}$ d'urée $(46 \% \mathrm{~N})$ à raison de $100 \mathrm{~kg} \cdot \mathrm{ha}^{-1}$ à début tallage et de $50 \mathrm{~kg} \cdot \mathrm{ha}^{-1}$ à début épiaison. Des diguettes anti-érosives ont été aménagées tout autour des essais pour limiter le ruissellement de l'eau de pluie au sein des parcelles.

Données climatiques: Une station météorologique automatique CIMEL ENERCO 405 (@ Cimel Electronique S.A.S France) a été installée le 13 juillet 2013 sur le site des essais pour l'enregistrement des données climatiques journalières suivantes: températures minimales et maximales, humidités relatives minimales, maximales et moyennes, radiations globales, vitesses moyennes du vent et hauteurs de pluie. Cependant, seules les données se rapportant aux températures et à la pluviométrie seront exploitées dans le cadre de ce travail. Du fait de l'installation de la station météorologique après le début des pluies de l'année 2013, les premières données climatiques de cette même année n'ont pu être enregistrées. Les données pluviométriques ont été néanmoins complétées grâce aux valeurs enregistrées à partir d'un pluviomètre à lecture directe installé sur le même site depuis le mois de mai 2013. Les dates de début et de fin de la saison des pluies ont été déterminées suivant les critères de Sivakumar (1988). Selon cet auteur, la date de début de la saison pluvieuse correspond au premier jour après le $1^{\text {er }}$ mai où la pluie cumulée avec celle des deux jours précédents atteint une hauteur minimale de $20 \mathrm{~mm}$ sans qu'il soit suivi pendant les 30 jours suivants d'une pause pluviométrie de plus de 7 jours. La date de fin de la saison des pluies correspond quant à elle au jour après le $1^{\text {er }}$ septembre à partir duquel il n'y a plus de pluie utile pendant deux décades. La différence entre les dates de fin et de début de la saison des pluies permet d'obtenir sa durée. Les périodes de déficit hydrique correspondent aux jours, pendant la saison pluvieuse, au cours desquels la pluviosité journalière n'atteint pas $5 \mathrm{~mm}$ (Sivakumar, 1988 ; Peugeot, 1995 ; Balme et al., 2005). Ainsi, une pluviométrie pentadaire est considérée déficitaire lorsque la hauteur de pluie enregistrée sur la pentade est inférieure à $25 \mathrm{~mm}$.

Paramètres agronomiques mesurés et calculés : Les paramètres agronomiques suivants ont été mesurés ou calculés au cours des différents essais : la durée de cycle semis-floraison (DSF), la durée de cycle semis- maturité (DSM), la hauteur des plants à la récolte (HAUT), le nombre total de talles par mètre carré à la récolte (TAL), le nombre de panicules par mètre carré à la récolte (PANI), le taux de talles infertiles (INFERT), le rendement grains (RDTG), le rendement paille (RDTP), l'indice de récolte (IR), le nombre d'épillets par panicule (EPIL), le nombre d'épillets vides par panicule (EPV), le taux de stérilité (STERIL) et le poids de mille grains (PMG).

Durée de cycle : 6 poquets situés aux abords du carré de rendement ont été retenus pour l'observation des stades floraison et maturité. Ces stades phénologiques ont été déterminés sur la base de l'échelle BBCH qui stipule que le stade phénologique d'une plante est celui atteint par sa tige principale (BBCH, 2001 ; Witzenberger et al., 1989 ; Lancashire et al., 1991). Dans le cadre de cette étude, la durée de cycle semis-floraison est le temps qu'il faut, après le semis, pour atteindre la floraison de $50 \%$ des poquets observés. Le stade maturité est atteint lorsque les grains des panicules sont durs et difficiles à couper en deux avec l'ongle (code 89 de l'échelle BBCH). Les durées de cycle semis-floraison et semis-maturité ont été exprimées en somme de températures avec une température de base de $13{ }^{\circ} \mathrm{C}$ (Angladette, 1966).

Hauteur des plants: La mesure de hauteur a été effectuée sur les six poquets retenus pour l'observation des stades phénologiques. La hauteur (en $\mathrm{cm}$ ) correspondant à une parcelle est la moyenne des mesures effectuées sur les six poquets depuis la base des plants jusqu'au sommet de la plus haute panicule.

Rendement et indice de récolte : Pour tous les essais, les 64 poquets centraux (8 lignes de 8 poquets) des parcelles élémentaires ont été retenus pour former le carré de rendement qui avait alors une superficie de 3,2 $\mathrm{m}^{2}$. Les grains de riz ont été récoltés à maturité et la production du carré de rendement a été pesée ainsi que la biomasse aérienne correspondante. Cette biomasse était constituée de talles, de feuilles et de panicules égrenées. Les grains et la biomasse ont été suffisamment pré-séchés à l'air en serre avant un séjour final à l'étuve à $65^{\circ} \mathrm{C}$ pendant une durée de $72 \mathrm{~h}$. Les taux d'humidité des organes ont été déterminés suite à ce séchage; $c e$ qui a permis de calculer le rendement grains en $\mathrm{kg} / \mathrm{ha}$ ajusté à 14\% d'humidité (RDTG) et le rendement paille (RDTP) (idem $\mathrm{kg} / \mathrm{ha}$ ). L'indice de récolte (IR) a été calculé suivant la formule ci-après : 


$$
I R=\frac{R D T G}{R D T G+R D T P}
$$

Composantes du rendement: A la récolte, on a procédé au comptage des poquets, talles et panicules du carré de rendement. Ce qui a permis de déterminer le nombre de talles au mètre carré (TAL), le nombre de panicules au mètre carré (PANI) et le taux de talles infertiles (INFERT).

$$
I N F E R T=100 \frac{T A L-P A N I}{T A L}
$$

En sus les panicules de cinq poquets du carré de rendement (choisis de façon aléatoire) ont été étudiées pour la détermination du poids de mille grains (PMG), du nombre total d'épillets par panicule (EPIL) et du nombre

\section{RÉSULTATS}

Données climatiques: En 2013, la température maximale journalière a oscillé entre $29{ }^{\circ} \mathrm{C}$ et $35^{\circ} \mathrm{C}$ depuis le semis du premier essai jusqu'au 11 septembre (le premier essai était à $51 \mathrm{JAS}$ et le second était à 37 JAS). La température maximale a ensuite varié autour de $35^{\circ} \mathrm{C}$ jusqu'à la fin des essais. La température minimale journalière a été plus stable et est généralement restée dans l'intervalle $20{ }^{\circ} \mathrm{C}-25{ }^{\circ} \mathrm{C}$ sauf à partir du 3 novembre (90 JAS au niveau deuxième essai) où des valeurs comprises entre $15{ }^{\circ} \mathrm{C}$ et $20{ }^{\circ} \mathrm{C}$ ont été enregistrées. Les températures de 2014 ont évolué dans les mêmes intervalles que celles de 2013 mais à partir du 22 septembre ( $60 \mathrm{JAS}$ et $45 \mathrm{JAS}$ respectivement pour les essais 1 et 2) les températures maximales journalières ont régulièrement pris des valeurs comprises entre $35^{\circ} \mathrm{C}$ et $40^{\circ} \mathrm{C}$ (Figure 1). En 2013, la saison des pluies a débuté le 3 juillet et l'on a enregistré une pluviosité annuelle de $738 \mathrm{~mm}$. Pendant cette même année, le premier essai a reçu $573 \mathrm{~mm}$ de pluie et le deuxième en a reçu $536 \mathrm{~mm}$. II a été dénombré 46 et 44 jours de pluie respectivement pour le premier et le deuxième essai. La répartition pentadaire de la pluviométrie a mis en évidence des périodes de déficit hydrique intra-saisonnier d'une durée cumulée de cinq pentades. Une sécheresse de fin de cycle a été observée à partir du 21 septembre après sept pentades consécutives humides. La sécheresse de fin de cycle est survenue alors que le premier essai était à $60 \mathrm{JAS}$ et le deuxième à $46 \mathrm{JAS}$. La saison des pluies a pris fin le 21 octobre, le premier essai était à alors à 91 JAS et le deuxième, à 77 JAS. En 2014, la saison des pluies a commencé le 29 juin et l'on a d'épillets vides par panicule (EPV). Le taux de stérilité (STERIL) a été calculé comme suit :

$$
S T E R I L=100 \frac{E P V}{E P I L}
$$

Traitement et analyse de données: Les analyses statiques ont été effectuées à l'aide du logiciel $R$ version 3.0.2 (R Core Team, 2013) sur toutes les variables excepté celles se rapportant à la phénologie (DSF et DSM). Le test de Shapiro-Wilk (Chapiro and Wilk, 1965) a permis de vérifier la distribution normale des données et le test de Bartlett (Bartlett, 1937) a été utilisé pour tester l'égalité des variances. Les analyses de variances ont été effectuées au seuil de significativité de $5 \%$. En cas de différence significative, les comparaisons multiples de moyennes ont été réalisées grâce au test de Tukey (Tukey, 1949).

enregistré une pluviosité annuelle de $693,5 \mathrm{~mm}$. Le premier essai de l'année 2014 a reçu $461 \mathrm{~mm}$ de pluie pendant 33 jours et le second en a reçu $377 \mathrm{~mm}$ pendant 31 jours. Les périodes de déficit hydrique au cours du cycle ont eu une durée cumulée de 8 pentades et la sécheresse de fin de cycle a été enregistrée à partir du 26 septembre après seulement trois pentades humides. Cette sécheresse de fin de cycle est survenue alors que les premier et deuxième essais étaient respectivement à 64 et 49 JAS. La saison des pluies a pris fin le 20 octobre soit à 88 JAS pour le premier essai et à 73 JAS pour le deuxième essai (Figure 1). En définitive, la saison des pluies a eu une durée de 110 jours en 2013 et de 113 jours en 2014.

Durée de cycle: Sur les deux années d'essais, les cycles des variétés n'ont pas été modifiés par la date de semis. En 2013, la floraison de NERICA 1 a eu lieu à 1040-1041 ${ }^{\circ} \mathrm{C}$ et celle de NERICA 4 est survenue à $1055-1056^{\circ} \mathrm{C}$. Les variétés à cycle plus court ont fleuri à 906-911 ${ }^{\circ} \mathrm{C}$ (NERICA 8) et à $925-926^{\circ} \mathrm{C}$ (NERICA 11). La durée de cycle semis-maturité correspondait à un temps thermique de $1471-1488^{\circ} \mathrm{C}$ (NERICA 1), 1500$1504^{\circ} \mathrm{C}$ (NERICA 4), $1379-1399^{\circ} \mathrm{C}$ (NERICA 8) et 1393$1416^{\circ} \mathrm{C}$ (NERICA 11). En 2014, les durées de cycle semis-floraison étaient de $1036-1049^{\circ} \mathrm{C}$ pour NERICA 1 et NERICA 4 et de $911-926{ }^{\circ} \mathrm{C}$ pour NERICA 8 et NERICA 11. Les durées de cycle semis-maturité étaient de $1460-1485^{\circ} \mathrm{C}$ pour NERICA 1 et NERICA 4 et de 1339-1347 ${ }^{\circ} \mathrm{C}$ pour NERICA 8 et NERICA 11.

Hauteur des plants à la récolte : $L$ a date de semis a eu un effet significatif sur la hauteur des plants à la récolte 


\section{Kouakou et al. J. Appl. Biosci. 2016 Performances agronomiques de quatre variétés de riz pluvial NERICA de plateau semées à différentes dates en zone soudano-sahélienne au Sénégal}

en 2013. Les plants avaient une hauteur moyenne de 92 $\mathrm{cm}$ avec le premier essai contre $82 \mathrm{~cm}$ avec le deuxième essai (tableau 1). Même si les plants étaient plus courts avec la deuxième date de semis en 2014, cette différence de hauteur par rapport à la première date n'était pas significative (tableau 2). Aucun effet variétal ni longueur de cycle n'a été observé sur les hauteurs des plants pendant ces deux années d'essais.

\section{Rendement et indice de récolte}

Rendement : Le rendement grains a significativement baissé de la première date à la deuxième date de semis sur les deux années. Cette baisse de rendement était de $75 \%$ (de $1792 \mathrm{~kg}$ ha $^{-1}$ à $439 \mathrm{~kg} \cdot \mathrm{ha}^{-1}$ ) en 2013 et de $79 \%$ (de 1047 kg.ha-1 à 220 kg.ha-1) en 2014 (tableaux 1 et 2). Aucune différence significative n'a été observée entre les rendements grains des quatre variétés sur les deux années. Les variétés à cycle court avaient cependant un rendement grains supérieur à celui des variétés tardives en 2013. Au niveau du rendement paille, aucun effet date de semis ni variété ni longueur de cycle n'a été noté sur les deux années d'essais. Ce rendement paille était en moyenne de $2605 \mathrm{~kg}$.ha-1 en 2013 et de $5336 \mathrm{~kg}^{-h^{-1}}$ en 2014 (tableaux 1 et 2).

Indice de récolte: Une différence significative a été notée au niveau de l'indice de récolte des dates de semis sur les deux années. Cet indice est passé de 0,39 à 0,15 en 2013 et de 0,16 à 0,04 en 2014 (tableaux 1 et 2). L'indice de récolte de la deuxième date de semis a donc connu une baisse de $63 \%$ et de $75 \%$ par rapport à la première date de semis respectivement en 2013 et en 2014. Par ailleurs, une différence significative a été observée entre les indices de récolte des variétés en 2013. Avec IR=0,36, NERICA 11 avait le meilleur indice de récolte suivis de NERICA $8(0,29)$ et de NERICA 4 $(0,23)$. NERICA 1 avait le plus petit indice de récolte, 0,19 (tableau 1). Sur les deux années expérimentales, l'indice de récolte des variétés précoces étaient supérieur à celui des variétés tardives (tableaux 1 et 2 ).

\section{Composantes du rendement}

Nombre de talles : En 2013, la date de semis et le cycle des variétés n'ont pas eu d'effet significatif sur le nombre de talles produit par unité de surface. En 2014, le nombre de talles a diminué de $22 \%$ de la première à la deuxième date de semis en passant de 162 talles.m-2 à 127 talles. $\mathrm{m}^{-2}$ sans toutefois varier en fonction du cycle des variétés étudiées. Sur les deux années, il a été noté une différence significative entre les talles produites par les variétés de riz. Ainsi, en 2003, NERICA 1 a produit le plus de talles ( 175 talles. $\mathrm{m}^{-2}$ ) suivi des trois autres variétés qui avaient un nombre similaire de talles (145 talles. $\mathrm{m}^{-2}$ en moyenne) (tableau 1). En 2014, NERICA 1 et NERICA 8 avaient produit en moyenne 157 talles. $\mathrm{m}^{-2}$ contre 131 talles. $\mathrm{m}^{-2}$ pour NERICA 11 et NERICA 4 (tableaux 1 et 2). Nombre de panicules: Le nombre de panicules produites a significativement baissé de la première date à la deuxième date de semis sur les deux années. Cette baisse était de $18 \%$ (de 132 panicules. $\mathrm{m}^{-2}$ à 108 panicules. $\mathrm{m}^{-2}$ ) en 2013 et de $26 \%$ (de 135 panicules. $\mathrm{m}^{-2}$ à 97 panicules. $\mathrm{m}^{-2}$ ) en 2014. Sur les deux années d'essais, le nombre de panicules produites ne différaient pas statistiquement entre les variétés prises individuellement ou regroupées par cycle (Tableaux 1 et 2).

Taux de talles infertiles: En 2013, le taux de talles infertiles a significativement augmenté de la première à la deuxième date de semis en passant de $10 \%$ à $31 \%$ (tableau 1). Par contre en 2014, la date de semis n'a pas eu d'effet significatif sur le taux de talles infertiles. Cependant un effet variétal a été noté sur ce taux pendant les deux années d'essais. En effet, NERICA 1 avait le taux le plus élevé (30\% en moyenne sur les deux années), NERICA 4 (18\% en moyenne sur les deux années) et NERICA 8 (16\% en moyenne sur les deux années) avaient des taux intermédiaires et NERICA 11 avait le plus faible taux de talles stériles (13\% en moyenne sur les deux années). Ce taux était plus élevé chez les variétés à cycle plus long en 2013 mais en 2014, il était statistiquement similaire quelque soit la longueur de cycle des variétés de riz (tableaux 1 et 2).

Nombre d'épillets par panicule : La date de semis a eu un effet significatif sur le nombre d'épillets produits par panicule en 2014. Le nombre d'épillets par panicule a baissé de $30 \%$ en passant de 108 à 76 de la première à la deuxième date de semis (tableau 2). La date de semis n'a eu aucun effet significatif sur le nombre d'épillets par panicule en 2013. Par contre en 2013, il a été noté un effet variétal sur ce paramètre. II était en moyenne de $174,120,85$ et 72 épillets par panicule respectivement pour NERICA 8, NERICA 11, NERICA 1 et NERICA 4 (tableau 1). En 2014, aucun effet variétal n'a été noté pour le nombre d'épillets par panicule. Les variétés plus précoces avaient produit plus d'épillets que les plus tardives lors des essais de 2013 cependant aucune différence statistique n'a été observée entre le nombre d'épillets produit en 2014 entre variétés précoces et variétés tardives (tableaux 1 et 2).

Taux de stérilité : Le taux de stérilité a significativement augmenté de la première à la deuxième date de semis sur les deux années. Cette augmentation du taux de stérilité était de $76 \%$ (de $34 \%$ à 60\%) en 2013 et de $31 \%$ (de 68\% à 90\%) en 2014 (tableaux 1 et 2). Une différence 
\begin{tabular}{l}
$\begin{array}{l}\text { Kouakou et al. J. Appl. Biosci. } 2016 \text { Performances agronomiques de quatre variétés de riz pluvial NERICA } \\
\text { de plateau semées à différentes dates en zone soudano-sahélienne au Sénégal }\end{array}$ \\
\hline
\end{tabular}

significative a été notée entre les taux de stérilité des variétés en 2014 . Ce taux était de $86 \%$ pour NERICA 1 et NERICA 4 et de $73 \%$ et $71 \%$ respectivement pour NERICA 8 et NERICA 11. Aucune différence statistique n'a été observée sur les taux de stérilité des variétés de riz en 2013. En 2013, le taux de stérilité des variétés ne dépendait pas de la longueur de leur cycle mais en 2014, les variétés à cycle plus long ont enregistré un taux de stérilité supérieur à celui des variétés précoces (tableaux 1 et 2).

Poids de mille grains : Aucun effet date de semis ni variété ni longueur de cycle n'a été noté sur le poids de mille grains sur les deux années d'essais. Le poids de mille grains était en moyenne de $24 \mathrm{~g}$ en 2013 et de $21 \mathrm{~g}$ en 2014 (tableaux 1 et 2).

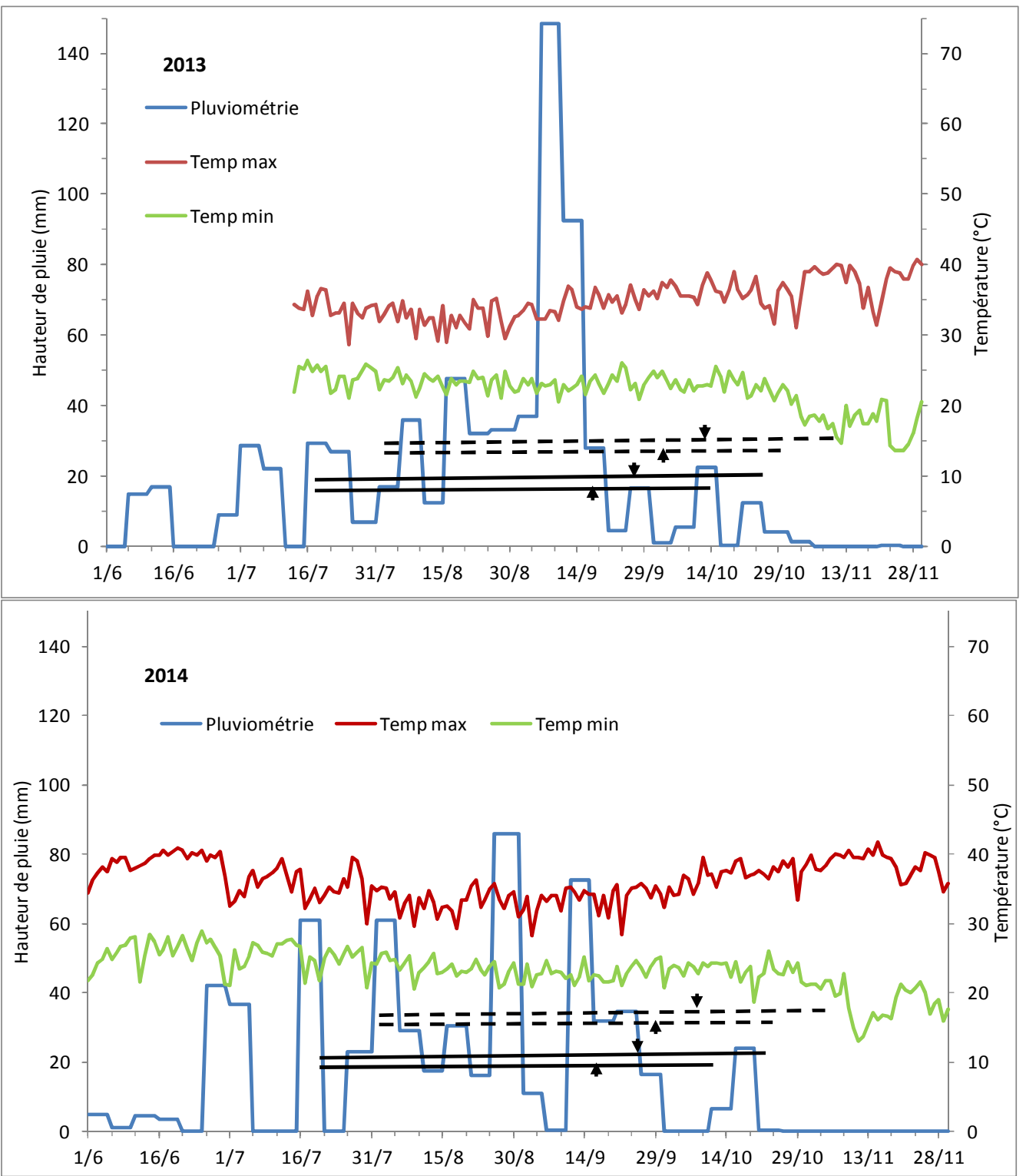

Figure 1: Températures maximales et minimales journalières et pluviométrie pentadaire enregistrées au cours des essais en 2013 et en 2014 avec positionnement des longueurs de cycle et de dates de floraison des variétés de riz. Les traits continus correspondent aux longueurs de cycle de la première date de semis et les traits discontinus correspondent aux longueurs de cycle de la deuxième date de semis. Les flèches matérialisent les périodes de floraison. 
Kouakou et al. J. Appl. Biosci. 2016 Performances agronomiques de quatre variétés de riz pluvial NERICA de plateau semées à différentes dates en zone soudano-sahélienne au Sénégal

Tableau 1: Valeurs moyennes des paramètres mesurés en 2013 suivant la date de semis, la variété, le cycle des variétés de riz et linteraction entre la date de semis et les autres facteurs

\begin{tabular}{|c|c|c|c|c|c|c|c|c|c|c|}
\hline & $\begin{array}{l}\text { HAUT } \\
(\mathrm{cm})\end{array}$ & $\begin{array}{c}\text { TAL } \\
\text { (talle/m2) } \\
\end{array}$ & $\begin{array}{c}\text { PANI } \\
\text { (panicule/m2) }\end{array}$ & $\begin{array}{c}\text { INFERT } \\
(\%)\end{array}$ & $\begin{array}{c}\text { RDTG } \\
\text { (kg/ha) }\end{array}$ & $\begin{array}{c}\text { RDTP } \\
\text { (kg/ha) }\end{array}$ & IR & EPIL & $\begin{array}{c}\text { STERIL } \\
(\%)\end{array}$ & $\begin{array}{c}\text { PMG } \\
\text { (g) }\end{array}$ \\
\hline \multicolumn{11}{|c|}{ Date de semis } \\
\hline Date1 & $92 \mathrm{a}$ & $147 a$ & $132 \mathrm{a}$ & $10.2 \mathrm{~b}$ & $1792 \mathrm{a}$ & $2764 a$ & $0.39 a$ & 118 a & $34 \mathrm{~b}$ & $23.8 \mathrm{a}$ \\
\hline Date2 & $82 \mathrm{~b}$ & 159 a & $108 \mathrm{~b}$ & $31.5 \mathrm{a}$ & $439 \mathrm{~b}$ & $2445 a$ & $0.15 b$ & $108 \mathrm{a}$ & $60 a$ & $24.4 \mathrm{a}$ \\
\hline \multicolumn{11}{|c|}{ Variété } \\
\hline NERICA1 & $81 \mathrm{a}$ & $175 \mathrm{a}$ & $117 \mathrm{a}$ & $31.4 \mathrm{a}$ & $775 \mathrm{a}$ & $2603 a$ & $0.19 \mathrm{c}$ & $85 b$ & $61.5 \mathrm{a}$ & $23.8 \mathrm{a}$ \\
\hline NERICA4 & $87 a$ & $145 b$ & 109 a & $26.1 \mathrm{ab}$ & $1065 \mathrm{a}$ & $2968 \mathrm{a}$ & $0.23 \mathrm{bc}$ & $72 b$ & $44.8 \mathrm{a}$ & $24.1 \mathrm{a}$ \\
\hline NERICA8 & $91 \mathrm{a}$ & $148 \mathrm{~b}$ & $126 a$ & $14.3 \mathrm{bc}$ & $1157 a$ & $2387 a$ & $0.29 \mathrm{ab}$ & $174 \mathrm{a}$ & $39.9 a$ & $24.0 \mathrm{a}$ \\
\hline NERICA11 & $88 \mathrm{a}$ & $144 \mathrm{~b}$ & 127 a & $11.7 \mathrm{c}$ & $1466 \mathrm{a}$ & $2461 \mathrm{a}$ & $0.36 \mathrm{a}$ & $120 \mathrm{ab}$ & $41.9 \mathrm{a}$ & $24.4 \mathrm{a}$ \\
\hline \multicolumn{11}{|c|}{ Longueur de cycle } \\
\hline MOYEN & $84 a$ & $160 \mathrm{a}$ & $113 \mathrm{a}$ & $28.7 \mathrm{a}$ & $920 \mathrm{~b}$ & $2785 a$ & $0.21 \mathrm{~b}$ & $79 b$ & $53.1 \mathrm{a}$ & $23.9 a$ \\
\hline COURT & $90 \mathrm{a}$ & $146 \mathrm{a}$ & 127 a & $13 \mathrm{~b}$ & $1311 \mathrm{a}$ & $2424 \mathrm{a}$ & $0.33 \mathrm{a}$ & $147 \mathrm{a}$ & $40.9 \mathrm{a}$ & $24.2 \mathrm{a}$ \\
\hline \multicolumn{11}{|c|}{ Date de semis $x$ Variété } \\
\hline Date 1 - NERICA1 & $82 \mathrm{a}$ & $154 a b$ & $130 \mathrm{a}$ & $15.5 \mathrm{c}$ & $1425 a b$ & $3101 \mathrm{a}$ & $0.31 a b c$ & $93 a b$ & $34.9 \mathrm{bc}$ & $23.1 \mathrm{a}$ \\
\hline Date 1 - NERICA4 & $91 \mathrm{a}$ & $150 a b$ & $133 \mathrm{a}$ & $11.9 \mathrm{c}$ & 1874 a & $3012 \mathrm{a}$ & $0.38 a b$ & $81 \mathrm{~b}$ & $16.0 \mathrm{c}$ & $23.9 \mathrm{a}$ \\
\hline Date 1 - NERICA8 & $99 \mathrm{a}$ & $143 a b$ & $134 \mathrm{a}$ & $6.4 c$ & $1906 \mathrm{a}$ & $2551 \mathrm{a}$ & $0.42 \mathrm{ab}$ & $133 a b$ & $37.0 \mathrm{bc}$ & $24.0 \mathrm{a}$ \\
\hline Date 1 - NERICA11 & 94 a & $140 \mathrm{~b}$ & $130 \mathrm{a}$ & $7.1 \mathrm{c}$ & $1962 \mathrm{a}$ & $2393 a$ & $0.45 a$ & $165 a b$ & $48.3 \mathrm{abc}$ & $23.9 \mathrm{a}$ \\
\hline Date 2 - NERICA1 & $79 \mathrm{a}$ & $196 a$ & $103 a$ & $47.2 \mathrm{a}$ & $124 \mathrm{c}$ & $2104 \mathrm{a}$ & $0.07 \mathrm{~d}$ & $78 \mathrm{~b}$ & $88.1 \mathrm{a}$ & $24.4 \mathrm{a}$ \\
\hline Date 2 - NERICA4 & $83 a$ & $139 \mathrm{~b}$ & $85 \mathrm{a}$ & $40.2 \mathrm{ab}$ & $256 \mathrm{c}$ & 2924 a & $0.07 \mathrm{~d}$ & $64 \mathrm{~b}$ & $73.5 \mathrm{ab}$ & $24.3 \mathrm{a}$ \\
\hline Date 2 - NERICA8 & $83 a$ & $152 a b$ & $119 \mathrm{a}$ & $22.3 \mathrm{bc}$ & 408 bc & $2222 a$ & $0.16 \mathrm{~cd}$ & $214 a$ & $42.8 \mathrm{abc}$ & $24.0 \mathrm{a}$ \\
\hline Date 2 - NERICA11 & $82 \mathrm{a}$ & $148 a b$ & $124 \mathrm{a}$ & $16.2 \mathrm{bc}$ & $969 a b c$ & $2530 \mathrm{a}$ & $0.28 b c$ & $76 b$ & $35.5 b c$ & $24.8 \mathrm{a}$ \\
\hline \multicolumn{11}{|c|}{ Date de semis $x$ longueur de cycle } \\
\hline Date1-MOYEN & $87 \mathrm{a}$ & $152 \mathrm{a}$ & $132 \mathrm{a}$ & $13.7 \mathrm{~b}$ & 1649 a & 3057 a & $0.35 \mathrm{~b}$ & $87 a$ & $25.4 \mathrm{~b}$ & $23.5 \mathrm{a}$ \\
\hline Date1-COURT & $97 a$ & $142 \mathrm{a}$ & $132 \mathrm{a}$ & $6.8 \mathrm{~b}$ & $1934 \mathrm{a}$ & $2472 a$ & $0.43 a$ & $149 \mathrm{a}$ & $42.7 \mathrm{~b}$ & $24.0 \mathrm{a}$ \\
\hline Date2-MOYEN & $81 \mathrm{~b}$ & $168 \mathrm{a}$ & $94 \mathrm{a}$ & $43.7 \mathrm{a}$ & $190 \mathrm{~b}$ & $2514 \mathrm{a}$ & $0.07 c$ & 71 a & $80.8 \mathrm{a}$ & $24.3 \mathrm{a}$ \\
\hline Date2-COURT & $82 \mathrm{~b}$ & $150 \mathrm{a}$ & $121 \mathrm{a}$ & $19.2 \mathrm{~b}$ & $689 \mathrm{~b}$ & $2376 a$ & $0.22 b$ & $145 \mathrm{a}$ & $39.2 \mathrm{~b}$ & $24.4 \mathrm{a}$ \\
\hline
\end{tabular}

Au niveau de chaque facteur, les moyennes en colonne affectées de la même lettre ne diffèrent pas significativement au seuil de $5 \%$ 
Kouakou et al. J. Appl. Biosci. 2016 Performances agronomiques de quatre variétés de riz pluvial NERICA de plateau semées à différentes dates en zone soudano-sahélienne au Sénégal

Tableau 2 : Valeurs moyennes des paramètres mesurés en 2014 suivant la date de semis, la variété, le cycle des variétés de riz et linteraction entre la date de semis et les autres facteurs

\begin{tabular}{|c|c|c|c|c|c|c|c|c|c|c|}
\hline & $\begin{array}{l}\text { HAUT } \\
(\mathrm{cm})\end{array}$ & $\begin{array}{c}\text { TAL } \\
\text { (talle/m2) }\end{array}$ & $\begin{array}{c}\text { PANI } \\
\text { (panicule/m2) }\end{array}$ & $\begin{array}{l}\text { INFERT } \\
(\%)\end{array}$ & $\begin{array}{c}\text { RDTG } \\
\text { (kg/ha) }\end{array}$ & $\begin{array}{l}\text { RDTP } \\
\text { (kg/ha) }\end{array}$ & IR & EPIL & $\begin{array}{c}\text { STERIL } \\
(\%)\end{array}$ & $\begin{array}{c}\text { PMG } \\
\text { (g) }\end{array}$ \\
\hline \multicolumn{11}{|c|}{ Date de semis } \\
\hline Date1 & $72 \mathrm{a}$ & $162 \mathrm{a}$ & $134.5 \mathrm{a}$ & $16.35 \mathrm{a}$ & $1047 a$ & 5558 a & $0.16 \mathrm{a}$ & $108 \mathrm{a}$ & $68 \mathrm{~b}$ & $20 \mathrm{a}$ \\
\hline Date2 & $67 a$ & $126 \mathrm{~b}$ & $99.6 \mathrm{~b}$ & $20.17 \mathrm{a}$ & $220 \mathrm{~b}$ & $5113 \mathrm{a}$ & $0.04 \mathrm{~b}$ & $76 b$ & $90 \mathrm{a}$ & $21 \mathrm{a}$ \\
\hline \multicolumn{11}{|c|}{ Variété } \\
\hline NERICA1 & $68 \mathrm{a}$ & $157 \mathrm{a}$ & $109.5 \mathrm{a}$ & $30.04 a$ & $642 \mathrm{a}$ & 5584 a & $0.09 \mathrm{a}$ & $87 a$ & 86 a & $22 \mathrm{a}$ \\
\hline NERICA4 & $68 \mathrm{a}$ & $130 \mathrm{~b}$ & 116.9 a & $10.94 \mathrm{~b}$ & 544 a & 5889 a & $0.08 \mathrm{a}$ & 85 a & $86 a$ & 19 a \\
\hline NERICA8 & $72 \mathrm{a}$ & $158 \mathrm{a}$ & $128.0 \mathrm{a}$ & $17.94 \mathrm{~b}$ & 683 a & $4572 \mathrm{a}$ & $0.12 \mathrm{a}$ & $92 \mathrm{a}$ & $73 b$ & $20 \mathrm{a}$ \\
\hline NERICA11 & $71 \mathrm{a}$ & $133 \mathrm{~b}$ & 113.9 a & $14.13 b$ & 665 a & 5298 a & $0.10 \mathrm{a}$ & $103 \mathrm{a}$ & $71 b$ & $19 \mathrm{a}$ \\
\hline \multicolumn{11}{|c|}{ Longueur de cycle } \\
\hline MOYEN & $68 \mathrm{a}$ & $144 \mathrm{a}$ & $113 \mathrm{a}$ & $20.5 \mathrm{a}$ & $593 a$ & $5736 a$ & $0.08 \mathrm{~b}$ & $86 a$ & $86 a$ & $21 \mathrm{a}$ \\
\hline COURT & $71 \mathrm{a}$ & $145 \mathrm{a}$ & $121 \mathrm{a}$ & $16.0 \mathrm{a}$ & 674 a & $4935 \mathrm{a}$ & $0.11 \mathrm{a}$ & 98 a & $72 b$ & $19 a$ \\
\hline \multicolumn{11}{|c|}{ Date de semis x Variété } \\
\hline Date 1 - NERICA1 & $70 \mathrm{a}$ & $169 a b$ & $113 \mathrm{bc}$ & $33.2 \mathrm{a}$ & $1159 \mathrm{a}$ & 5821 a & $0.16 \mathrm{a}$ & $90 \mathrm{a}$ & $78.5 \mathrm{a}$ & $21.7 \mathrm{a}$ \\
\hline Date 1 - NERICA4 & $68 \mathrm{a}$ & $164 a b$ & $150 \mathrm{a}$ & $8.4 \mathrm{~cd}$ & $945 a b c$ & $6104 a$ & $0.13 a b c$ & $100 \mathrm{a}$ & $74.2 \mathrm{a}$ & $18.1 \mathrm{a}$ \\
\hline Date 1 - NERICA8 & $77 \mathrm{a}$ & $183 \mathrm{a}$ & $145 a$ & $20.5 a b c$ & $1112 a b$ & 4446 a & $0.20 \mathrm{a}$ & 118 a & 59.9 a & $21.6 \mathrm{a}$ \\
\hline Date 1 - NERICA11 & $74 \mathrm{a}$ & $134 \mathrm{~b}$ & $130 a b$ & $3.4 \mathrm{~d}$ & $971 a b c$ & $5863 \mathrm{a}$ & $0.14 a b$ & $125 \mathrm{a}$ & $60.8 \mathrm{ab}$ & $16.9 \mathrm{a}$ \\
\hline Date 2 - NERICA1 & $66 \mathrm{a}$ & $146 \mathrm{ab}$ & $106 \mathrm{bc}$ & $26.9 \mathrm{ab}$ & $124 \mathrm{~d}$ & $5346 a$ & $0.02 \mathrm{~d}$ & $85 a$ & $93.0 \mathrm{ab}$ & $22.2 \mathrm{a}$ \\
\hline Date 2 - NERICA4 & $67 a$ & $97 c$ & $84 c$ & $13.5 \mathrm{bcd}$ & $144 \mathrm{~d}$ & $5674 \mathrm{a}$ & $0.03 d$ & 69 a & $97.6 \mathrm{ab}$ & $20.6 \mathrm{a}$ \\
\hline Date 2 - NERICA8 & 66 a & $133 \mathrm{~b}$ & $111 b c$ & $15.4 \mathrm{bcd}$ & $255 \mathrm{~cd}$ & 4698 a & $0.05 \mathrm{~cd}$ & $67 a$ & $86.9 \mathrm{~b}$ & $17.7 \mathrm{a}$ \\
\hline Date 2 - NERICA11 & $67 a$ & $132 b$ & $98 c$ & $24.9 a b$ & $358 \mathrm{bcd}$ & $4734 a$ & $0.07 \mathrm{bcd}$ & $82 \mathrm{a}$ & $81.6 \mathrm{~b}$ & $21.7 \mathrm{a}$ \\
\hline \multicolumn{11}{|c|}{ Date de semis $x$ longueur de cycle } \\
\hline Date1-MOYEN & $69 a$ & $166 \mathrm{a}$ & $132 \mathrm{a}$ & $20.8 a$ & $1052 \mathrm{a}$ & $5963 \mathrm{a}$ & $0.15 \mathrm{a}$ & $95 a b$ & $76 b$ & $20 a$ \\
\hline Date1-COURT & 75 a & 158 a & $137 \mathrm{a}$ & $11.9 \mathrm{a}$ & $1041 \mathrm{a}$ & 5154 a & $0.17 \mathrm{a}$ & $121 \mathrm{a}$ & $60 c$ & $19 \mathrm{a}$ \\
\hline Date2-MOYEN & $67 \mathrm{a}$ & $121 \mathrm{a}$ & $95 \mathrm{~b}$ & $20.2 \mathrm{a}$ & $134 \mathrm{~b}$ & $5510 \mathrm{a}$ & $0.02 b$ & $77 b$ & $95 \mathrm{a}$ & $21 \mathrm{a}$ \\
\hline Date2-COURT & $67 a$ & $132 \mathrm{a}$ & $105 b$ & $20.2 \mathrm{a}$ & $306 b$ & $4716 a$ & $0.06 \mathrm{~b}$ & $74 \mathrm{~b}$ & $84 a b$ & $20 a$ \\
\hline
\end{tabular}

Au niveau de chaque facteur, les moyennes en colonne affectées de la même lettre ne diffèrent pas significativement au seuil de $5 \%$. 


\section{Kouakou et al. J. Appl. Biosci. 2016 Performances agronomiques de quatre variétés de riz pluvial NERICA}

de plateau semées à différentes dates en zone soudano-sahélienne au Sénégal

\section{DISCUSSION}

Les expérimentations ont été conduites sur la même période de la saison pluvieuse puisque sur les deux années, les premiers semis ont été effectués pendant la cinquième pentade du mois de juillet et les seconds pendant la deuxième pentade du mois d'août. Sur les deux années, la fin de la saison pluvieuse n'a pas varié et elle a été observée pendant la cinquième pendate du mois d'octobre. Cette caractéristique de la fin de la saison des pluies a été mise en évidence lors d'autres travaux portant sur la zone soudano-sahélienne (Balme et al., 2005; NDong, 2003; Sarr et al., 2011) . Les températures enregistrées au cours de ces deux années d'essais ont eu des évolutions similaires et sont restées dans les mêmes ordres de grandeur quelque soit la date de semis. Ainsi, les sommes de températures nécessaires à la mise à fleur des variétés d'une part et à leur maturité d'autre part ont été proches pour une longueur de cycle donné. Les périodes de floraison (de maturité) des variétés de même cycle ne pouvaient alors pas différer puisque tous les plants ont été soumis aux mêmes conduites agronomiques. C'est pour cela que les variétés précoces NERICA 8 et NERICA 11 ont fleuri (atteint la maturité) aux mêmes périodes indépendamment de la date de semis et que les variétés à cycle plus long, NERICA 1 et NERICA 4 ont fleuri (atteint la maturité) aux mêmes dates. La différence variétale observée dans les périodes de mise à fleur (de maturité) est imputable à la différence naturelle dans la longueur des cycles des variétés étudiées (AfricaRice, 2008). Les températures enregistrées au cours des quatre essais étaient favorables à une bonne croissance et un bon développement des plants (Angladette, 1966). Cependant, les expérimentations de l'année 2014 ont été moins arrosées que celles de l'année 2013. En effet, le premier essai de 2014 a reçu $112 \mathrm{~mm}$ de pluie de moins que celui de 2013. De même, le deuxième essai de 2014 a reçu $159 \mathrm{~mm}$ de pluie de moins que celui de 2013. Le premier essai de 2014 a même reçu une hauteur de pluie déficitaire de $75 \mathrm{~mm}$ par rapport au dernier essai de 2013. Il a été enregistré moins de jours de pluies en 2014 qu'en 2013. Les périodes de déficit hydrique intrasaisonnier de la deuxième année d'essai ont été plus nombreuses et plus longues que celles de la première année. Ce climat plus sec de l'année 2014 a eu un effet négatif sur les paramètres agronomiques suivants : la hauteur, le rendement grains, le rendement paille, l'indice de récolte et le taux de stérilité des variétés de riz testées. En effet, la hauteur de ces variétés a diminué de $20 \mathrm{~cm}$ en passant de 81-91 cm en 2013 à 68-72 cm en
2014. Les rendements grains de NERICA 4, NERICA 8 et NERICA 11 ont diminué de moitié en passant de 1065 1466 kg.ha-1 en 2013 à 544-665 kg.ha-1 en 2014. La diminution de rendement grains chez NERICA 1 était d'importance moindre (17\%) puisse qu'il est passé de $775 \mathrm{~kg} \cdot \mathrm{ha}^{-1}$ à $642 \mathrm{~kg} \cdot$ ha $^{-1}$. Le développement végétatif a été plus abondant chez les variétés de riz étudiées lors de la deuxième année d'essai puisque les rendements paille et l'indice de récolte représentaient respectivement le double et la moitié de ceux de l'année 2013 (tableaux 1 et 2). Le taux de stérilité des variétés a connu une augmentation de 40 à $92 \%$ en passant de $40-62 \%$ en 2013 à $71-86 \%$ en 2014. Ces taux de stérilité étaient plus élevés chez les variétés à cycle plus long (NERICA 1 et NERICA 4) que chez les variétés à cycle plus court (NERICA 8 et NERICA 11) : $53 \%$ contre $41 \%$ en 2013 et $86 \%$ contre $72 \%$ en 2014 . La longueur de cycle n'a eu d'effet significatif que sur le rendement grains et le nombre d'épillets par panicule en 2013. Puisse qu'en cette même année, le facteur variété n'a pas eu d'effet significatif sur le rendement grains, le plus fort rendement grains obtenu chez les variétés plus précoces est essentiellement dû au fait qu'elles avaient produit plus d'épillets par panicule. En 2014, la longueur de cycle n'a eu d'effet significatif que sur l'indice de récolte et le taux de stérilité. Cela met en évidence que les rendements grains enregistrés sur chacune des deux années étaient largement tributaires de paramètres agronomiques élaborés pendant la phase reproductive des variétés. Or sur les deux années, les conditions climatiques de fin de cycle (pour les premiers essais) et de milieu et fin de cycle (pour les deuxièmes essais) ont été plus sèches. Cela s'est traduit par des températures maximales journalières de plus en plus élevées et des périodes de déficit hydrique. Les variétés à cycle plus court des premières dates de semis ont fleurit avant la survenue de cette période sèche. Cependant, la floraison des variétés à cycle plus long des premiers semis a été partiellement affectée par cette période de déficit hydrique. Les essais des deuxièmes dates de semis ont été les plus exposés à ce climat sec de la fin de la saison pluvieuse puisque cette période était intervenue avant la mise à fleur des variétés quelque soit la longueur de leur cycle. Cette raison explique alors les forts taux de stérilité des épillets enregistrés d'une part chez les variétés à cycle plus long et d'autre part sur les essais semés tardivement tant en 2013 qu'en 2014 (Lacharme, 2001). En effet, la phase reproductive est la phase la plus sensible du cycle cultural du riz et un déficit hydrique enregistré pendant 


\section{Kouakou et al. J. Appl. Biosci. 2016 Performances agronomiques de quatre variétés de riz pluvial NERICA de plateau semées à différentes dates en zone soudano-sahélienne au Sénégal}

cette phase compromet largement les rendements. II est alors logique que les rendements grains aient baissé de l'ordre de 50 à $90 \%$ sur les essais de la deuxième date de semis comparativement à ceux des premières dates de semis. Le fait que le rendement paille ne soit pas dépendant de la date de semis ni du cycle des variétés s'explique par le fait que le stress climatique était survenu au moment où l'essentiel de la biomasse (talles et feuilles essentiellement) des variétés avait été élaborée (AfricaRice, 2008). Les organes de riz élaborés pendant la phase reproductive ont été les plus soumis à l'effet régressif du déficit hydrique : la formation des panicules et la fécondation des épillets (Lacharme, 2001). Le fait

\section{CONCLUSION}

Les essais avaient pour objectif d'étudier l'effet de la date de semis sur les performances agronomiques de quatre variétés de riz pluvial de plateau de type NERICA. Le choix porté sur les NERICA se justifiait par le fait que ces variétés de riz se développent bien dans des environnements à faible ressource hydrique comme la zone soudano-sahélienne. II ressort que la saison des pluies a eu une durée moyenne de 110 jours et que la gamme de températures a été favorable au bon développement des variétés de riz étudiées. Les cycles les plus longs de ces variétés étant de 100 jours, le semis des essais aurait dû se faire au plus tard 10 jours après le début de la saison des pluies. Des paramètres climatiques suivis pendant les essais, la répartition de la pluviométrie a été le facteur limitant dans l'expression des performances agronomiques des variétés étudiées. La saison des pluies, sur les deux années, a été en effet marquée par des périodes de déficit hydrique intra-

\section{REMERCIEMENTS}

Le premier auteur est reconnaissant à la Coopération allemande DAAD (Deutscher Akademischer Austauschdienst) qui lui a accordé une bourse pour la préparation de sa thèse de doctorat. Les travaux de terrain ont été financés par AfricaRice à travers le Projet

\section{RÉFÉRENCES}

ADRAO, 2008. Guide pratique de la culture des NERICA de plateau 2008. Cotonou, Bénin : Centre du riz pour l'Afrique (ADRAO). $36 \mathrm{p}$.

AfricaRice, 2008. NERICA: the New Rice for Africa - a Compendium. E A Somado, R G Guei and SO Keya (eds.). Cotonou, Benin : Africa Rice Center (WARDA); Rome, Italy: FAO; Tokyo, Japan: Sasakawa Africa Association. 210 p. que les poids de mille grains n'étaient pas fonction de la variété, de la longueur de cycle et de la date de semis est dû au fait que les quatre variétés étudiées ne différaient pas à l'origine par ce caractère (AfricaRice, 2008) mais aussi par le fait que les conditions climatiques qui prévalaient lors du remplissage des grains étaient similaires pour toutes les variétés dans tous les cas. L'effet négatif très prononcé de la sécheresse de fin de cycle aurait pu être atténué si les semis avaient été effectués un peu plus tôt, au plus tard 10 jours après le début de la saison des pluies puisque les plus longs cycles étaient de 100 jours et que la saison pluvieuse à eu une durée de 110-113 jours sur les deux années.

saisonnier et de fin de cycle. Les périodes de déficit hydrique de fin de cycle ont été les plus déterminantes puisqu'elles ont coïncidé avec les périodes de floraison des variétés de riz étudiées. Les paramètres agronomiques élaborés pendant les phases de reproduction et de maturation des variétés de riz ont été les plus sujets à l'effet régressif de ces périodes de déficit hydrique. Ces effets ont été plus prononcés sur les essais semés un peu plus tardivement puisque, par exemple, les rendements grains des essais des deuxièmes dates de semis ont baissé d'au moins 75\% par rapport à ceux des premières dates de semis. II ressort alors que les quatre variétés de riz NERICA pluvial étudiées peuvent certes être cultivées dans la zone soudano-sahélienne de Sinthiou Malème mais il faut préférer les variétés très précoces de 75-85 jours (NERICA 8 et NERICA 11) et les semer avant la cinquième pentade du mois de juillet pour ne pas compromettre les rendements grains.

CCAFS (Climate Change Agriculture and Food Security) et suivis au quotidien par Mamadou Lamine Sonko et M'Baye Sarr Diop. Diarétou Diop, Emile Agbangba et Thierry Tovignan ont contribué à l'écriture des lignes de commande pour les tests statistiques sous $\mathrm{R}$.

Angladette A., 1966. Le riz, G-P, édition Maisonneuve et Larose, Paris, France, $930 \mathrm{p}$.

APRAO, 2012. Projet Amélioration de la Production du Riz en Afrique de l'Ouest en Réponse à la Flambée des Prix des Denrées Alimentaires (GCP/RAF/453/SPA)/ Composante Sénégal. Version provisoire, mai 2012. Ministère de l'Agriculture. Dakar. Sénégal. 49 p. 
Kouakou et al. J. Appl. Biosci. 2016 Performances agronomiques de quatre variétés de riz pluvial NERICA de plateau semées à différentes dates en zone soudano-sahélienne au Sénégal

www.fao.org/.../APRAO_Etude

Capitalisation_Version provisoire_Mai

Arraudeau M. A. et Vergara B. S., 1992. Manuel illustré de riziculture pluvial, IRRI-IRAT,

Balme M., Galle S.et Lebel T., 2005. Démarrage de la saison des pluies au Sahel : variabilité aux échelles hydrologique et agronomique, analysée à partir des données EPSAT-Niger. Sécheresse $16(1): 15-22$

Bartlett, M. S. (1937). "Properties of sufficiency and statistical tests". Proceedings of the Royal Statistical Society, Series A 160, 268-282

$\mathrm{BBCH}, 2001$. Stades phénologiques des mono-et dicotylédones cultivées 2. Édition, 2001. Rédigé par Uwe Meier. Centre Fédéral de Recherches Biologiques pour l'Agriculture et les Forêts. 166 $\mathrm{p}$

Brüntrup M., Nguyen T. et Kaps C., 2006. Le marché du riz au Sénégal. In Agriculture et développement rural, 1, 2006. Pp 23-26. http://www.rural21.com/ uploads/media/ELR_Le_marche_du_riz_au_Sen egal_0106.pdf

Chopart J.L., 1980. Étude au champ des systèmes racinaires des principales cultures pluviales au Sénégal (Arachide, mil, sorgho, riz pluvial). Thèse de Doctorat. Institut National Polytechnique de Toulouse, Toulouse, France, $160 \mathrm{p}$.

Del Villar P. M., Bauer J-M., Maiga A., Ibrahim L., 2011.Crise rizicole, évolution des marchés et sécurité alimentaire en Afrique de l'Ouest. Ministère des Affaires étrangères. France. $61 p$

Dieng A., Sagna M., Babou M., Dione F., Diallo B., 2011. Analyse de la compétitivité du riz local au Sénégal. Programme de renforcement de la recherche sur la sécurité alimentaire en Afrique de l'Ouest. Résumé n¹-2011-12. $9 \mathrm{p}$.

ENDA DIAPOL, 2010. Implication des acteurs ruraux dans la définition des politiques de développement des filières vivrières en Afrique de l'Ouest et du Centre. Rapport de mission au Sénégal. Document provisoire. 64p. www.fidafrique.net//MG/pdf/Etude_Senegal.pdf

FAO, 2011. Aperçu du développement rizicole au Sénégal. Division de la production végétale et de la protection des plantes (AGP) de la FAO. Bureau Afrique de l'Ouest. Dakar. Sénégal. 10p

Kelly V., Reardon T., Fall A., Diagana B. N., 1998. Impact des politiques de prix et de revenus sur la consumation et l'offre des produits agricoles.
Volume 1 \& 2, partie I \& II. Rapport final du Projet ISRA/IFPRI.

Lancashire, P. D., H. Bleiholder, P. Langelüddecke, R. Stauss, T.Van Den Boom, E. Weber und A. Witzenberger, 1991 : An uniform decimal code for growth stages of crops and weeds. Ann. appl. Biol. 119, 561-601.

NDong J. B., 2003. Caractérisation de la saison des pluies dans le centre-ouest du Sénégal. Publication de l'Association Internationale de Climatologie 15 : 326-332

Peugeot C. 1995. Influence de l'encroûtement superficiel du sol sur le fonctionnement hydrologique d'un versant sahélien. Thèse de doctorat, Université de Grenoble, France, 305 p. + annexes.

PNAR, 2009. Stratégie Nationale de Développement de la Riziculture. Programme National d'autosuffisance en riz. Ministère de l'Agriculture. Dakar, Sénégal. $26 \mathrm{p}$.

R Core Team (2013). R: A language and environment for statistical computing. R Foundation for Statistical computing, Vienna, Austria. (http://www.Rproject.org).

Salack S., Muller B. et Gaye A. T., 2011. Rain-based factors of high agricultural impacts over Senegal. Part I: integration of local to subregional trends and variability. Theor Appl Climatol 106:1-22

Sarr B., Kafando L. et Atta S., 2011. Identification des risques climatiques de la culture du maïs au Burkina Faso. Int. J. Biol. Chem. Sci. 5(4): 16591675

Seck M., 2015. AutosuffisAnce en riz : $600000 \mathrm{t}$ de riz blanc attendues au Sénégal selon Macky Sall. In Agriinfos Mensuel d'informations Agricoles et rurales - 9e Année - $\mathrm{N}^{\circ} 81$ - Issn 0850-8844. Avril 2015. P 4. www.inter-reseaux.org/ IMG/pdf/AGRI_INFOS_No81_AVRIL_2015.pdf

Shapiro S. S. and M. B. Wilk, « An analysis of variance test for normality (complete samples)", Biometrika, vol. 52, no 3-4, 1965, p. 591-611 (DOI 10.1093/biomet/52.3-4.591, JSTOR 2333709)

Sié M., 1997. Analyse des contraintes photothermiques en vue de l'adaptation des variétés de riz irrigué au Sahel. Thèse de doctorat. École Nationale Supérieure Agronomique de Montpellier, Montpellier, France, $138 \mathrm{p}$.

Sivakumar MVK, 1988. Predicting rainy season potential from the onset of rains in Southern Sahelian and 
Kouakou et al. J. Appl. Biosci. 2016 Performances agronomiques de quatre variétés de riz pluvial NERICA de plateau semées à différentes dates en zone soudano-sahélienne au Sénégal

Sudanian climatic zones of West Africa. Agricultural.and Forest Meteorology 42 : 295305.

Tukey, John (1949). "Comparing Individual Means in the Analysis of Variance". Biometrics 5 (2): 99-114.
Witzenberger, A., H. Hack und T. Van Den Boom, 1989 : Erläuterungen zum BBCH Dezimal-Code für die Entwicklungsstadien des Getreides - mit Abbildungen. Gesunde Pflanzen 41, 384-388 